\title{
Arrays with Local Centers of Symmetry in Space Groups Pca21 and Pna21
}

By Richard E. Marsh and Verner Schomaker ${ }^{1}$

Beckman Institute, California Institute of Technology, Pasadena, California 91125, USA

and Frank H. Herbstein

Department of Chemistry, Technion - Israel Institute of Technology, Haifa, Israel 32000

\begin{abstract}
Of the several hundred structures in the Cambridge Structural Database (1992) having space groups Pca2 1 or $P n a 2_{1}$ and more than one molecule in the asymmetric unit $(Z>4)$, approximately three-quarters contain local centers of symmetry. These local centers, which are not crystallographic centers, occur predominantly near $\mathrm{x}$ $=1 / 8, \mathrm{y}=1 / 4$ in $P c a 21$ or near $\mathrm{x}=1 / 8, \mathrm{y}=0$ in $P n a 21$; this also holds for the limited number of examples we have examined of pseudo-centrosymmetric molecules with $Z=4$. Local centers at these points create unusual correlations between corresponding atoms in the two molecules.
\end{abstract}

\section{MATERIAL FOR DEPOSIT}

\footnotetext{
${ }^{1}$ Deceased
} 
Table 1. Space group $P c a 2{ }_{1}: 172$ examples of crystals (identified by their REFCODES) which have local centers of symmetry ( 169 with $Z=8$ and 3 with $Z=4$ ). As noted in the text, the mean $\mathrm{x}$ and y coordinates of the local centers have been adjusted to lie within the limits $0^{2} \mathrm{x}, \mathrm{y}^{2} 1 / 4$, with mean $\mathrm{z}=0$. 'rmsd' here, and in Table 2, means the "square root of the mean-square deviation" of the individual atoms from the local center.

\begin{tabular}{|c|c|c|c|c|c|c|}
\hline REFCODE & $\langle\mathrm{x}\rangle$ & rmsd & $\langle y\rangle$ & rmsd & $\langle\mathrm{z}\rangle(\mathrm{rmsd})$ & $\begin{array}{l}\text { No. of Atoms } \\
\text { and Remarks }\end{array}$ \\
\hline ALPHNI & 0.125 & 0.10 & 0.222 & 0.18 & 0.08 & 11 \\
\hline BETZLB10 & 0.127 & 0.08 & 0.244 & 0.41 & 0.07 & 24 \\
\hline BEZWAE & 0.103 & 0.05 & 0.011 & 0.06 & 0.06 & 14 \\
\hline BINDPY & 0.184 & 0.03 & 0.247 & 0.05 & 0.03 & 17 \\
\hline \multirow[t]{2}{*}{ BIRCUA } & 0.141 & 0.15 & 0.217 & 0.11 & 0.20 & 18 \\
\hline & 0.130 & 0.02 & 0.245 & 0.02 & 0.03 & 21 \\
\hline \multicolumn{7}{|l|}{ BIVBUD20 } \\
\hline BONCEM & 0.129 & 0.08 & 0.243 & 0.06 & 0.06 & 24 \\
\hline BTHCFE & 0.126 & 0.04 & 0.241 & 0.03 & 0.05 & 24 \\
\hline BUHPID & 0.122 & 0.13 & 0.246 & 0.08 & 0.14 & 37 \\
\hline BUNDOD & 0.127 & 0.09 & 0.244 & 0.08 & 0.07 & 24 \\
\hline BUNYIS & 0.171 & 0.14 & 0.242 & 0.22 & 0.16 & 43 \\
\hline BUPSEK & 0.123 & 0.05 & 0.236 & 0.02 & 0.06 & $16(a, b)$ \\
\hline BURWAM & 0.127 & 0.03 & 0.238 & 0.07 & 0.02 & 21 \\
\hline BUTNEJ & 0.119 & 0.04 & 0.248 & 0.03 & 0.05 & 25 \\
\hline BUYNAC & 0.115 & 0.01 & 0.250 & 0.01 & 0.01 & 6 \\
\hline BXCGPL & 0.036 & 0.17 & 0.250 & 0.17 & 0.06 & 22 \\
\hline BZAPUC20 & 0.130 & 0.02 & 0.248 & 0.04 & 0.02 & 20 \\
\hline CAFVAG & 0.125 & 0.01 & 0.241 & 0.00 & 0.01 & $6, Z=4$ \\
\hline CAYMAQ & 0.129 & 0.08 & 0.244 & 0.15 & 0.17 & 24 \\
\hline CGLCOB & 0.126 & 0.08 & 0.249 & 0.09 & 0.10 & 25 \\
\hline CIJVAS & 0.003 & 0.06 & 0.249 & 0.06 & 0.04 & 28 Pbca? \\
\hline \multirow[t]{2}{*}{ CIPCIN } & 0.123 & 0.28 & 0.234 & 0.22 & 0.13 & 19 \\
\hline & 0.131 & 0.08 & 0.006 & 0.06 & 0.01 & $14(c)$ \\
\hline \multicolumn{7}{|l|}{ CIWBOZ10 } \\
\hline & 0.036 & 0.19 & 0.250 & 0.01 & 0.20 & \\
\hline CMALAV & 0.170 & 0.52 & 0.141 & 0.52 & 0.26 & 23 \\
\hline \multirow[t]{2}{*}{ CNSCCO } & 0.208 & 0.02 & 0.249 & 0.02 & 0.07 & $21(\mathrm{c})$ \\
\hline & 0.124 & 0.09 & 0.084 & 0.06 & 0.07 & \\
\hline CRPPFE & 0.119 & 0.18 & 0.242 & 0.13 & 0.08 & 36 \\
\hline CTMFRD & 0.122 & 0.02 & 0.248 & 0.01 & 0.05 & 13 \\
\hline СТPHMI & 0.125 & 0.08 & 0.249 & 0.08 & 0.05 & 17 \\
\hline \multirow[t]{2}{*}{ CUCDIM } & 0.123 & 0.02 & 0.244 & 0.07 & 0.03 & 18 \\
\hline & 0.130 & 0.04 & 0.250 & 0.08 & 0.05 & 23 \\
\hline \multicolumn{7}{|l|}{ CUESAL02 } \\
\hline CXTGER & 0.122 & 0.04 & 0.247 & 0.05 & 0.03 & 10 \\
\hline DAZPEN & 0.124 & 0.05 & 0.237 & 0.02 & 0.08 & $6, Z=4$ \\
\hline DETPOH & 0.135 & 0.02 & 0.243 & 0.05 & 0.06 & 28 \\
\hline DEYNAP & 0.043 & 0.06 & 0.233 & 0.09 & 0.10 & 14 \\
\hline DIDHED & 0.120 & 0.04 & 0.245 & 0.06 & 0.04 & 35 \\
\hline DIXVOV & 0.001 & 0.05 & 0.250 & 0.05 & 0.06 & 18 Pbca? \\
\hline DOKKOD & 0.127 & 0.02 & 0.245 & 0.14 & 0.02 & 18 \\
\hline
\end{tabular}




\begin{tabular}{|c|c|c|c|c|c|c|}
\hline DOLYEI & 0.138 & 0.06 & 0.249 & 0.10 & 0.05 & $25(d)$ \\
\hline DUVTOD & 0.000 & 0.24 & 0.247 & 0.23 & 0.22 & 19 \\
\hline EPNPHD10 & 0.036 & 0.01 & 0.231 & 0.01 & 0.01 & $11, Z=4$. \\
\hline ESALSN & 0.113 & 0.05 & 0.241 & 0.08 & 0.03 & 23 \\
\hline ETUZNT & 0.108 & 0.03 & 0.247 & 0.07 & 0.03 & 18 \\
\hline FADKEA & 0.120 & 0.10 & 0.239 & 0.05 & 0.09 & $54(\mathrm{a})$ \\
\hline FAJWIW & 0.117 & 0.03 & 0.249 & 0.10 & 0.19 & 11 \\
\hline FAYLEW & 0.124 & 0.03 & 0.239 & 0.04 & 0.06 & 12 \\
\hline FECHOK & 0.004 & 0.21 & 0.245 & 0.28 & 0.11 & 15 \\
\hline FECTOW & 0.000 & 0.11 & 0.249 & 0.13 & 0.24 & 12 Pbca? \\
\hline FEGTIU & 0.130 & 0.03 & 0.237 & 0.11 & 0.03 & 25 \\
\hline FESWAB & 0.123 & 0.07 & 0.250 & 0.06 & 0.08 & 29 (a) \\
\hline FEZRUX & 0.200 & 0.13 & 0.231 & 0.05 & 0.21 & 18 \\
\hline FIDCEA & 0.116 & 0.03 & 0.250 & 0.01 & 0.02 & 36 \\
\hline FILNAP & 0.122 & 0.05 & 0.234 & 0.15 & 0.05 & 33 \\
\hline FIPYEI & 0.124 & 0.09 & 0.234 & 0.07 & 0.09 & 21 \\
\hline FOWYUL10 & 0.001 & 0.06 & 0.248 & 0.05 & 0.07 & 24 Pbca? \\
\hline FUHPAZ & 0.018 & 0.06 & 0.234 & 0.05 & 0.05 & 20 \\
\hline GAPWAV & 0.123 & 0.01 & 0.246 & 0.01 & 0.01 & 12 \\
\hline GATYUV & 0.123 & 0.04 & 0.245 & 0.07 & 0.06 & 35 \\
\hline GAYGUI & 0.073 & 0.16 & 0.249 & 0.07 & 0.11 & 26 \\
\hline GAZDOA & 0.124 & 0.04 & 0.250 & 0.04 & 0.05 & 18 \\
\hline GLTHRE & 0.122 & 0.03 & 0.245 & 0.06 & 0.03 & 13 \\
\hline HACFUM & 0.119 & 0.05 & 0.240 & 0.08 & 0.04 & 23 \\
\hline HACGAT & 0.110 & 0.04 & 0.246 & 0.04 & 0.05 & 17 \\
\hline HAFXOB & 0.135 & 0.04 & 0.249 & 0.04 & 0.05 & 35 \\
\hline HAZNAX & 0.126 & 0.11 & 0.245 & 0.10 & 0.10 & 22 \\
\hline HEMCOR & 0.125 & 0.09 & 0.222 & 0.12 & 0.12 & 45 \\
\hline HIDSIW & 0.129 & 0.08 & 0.242 & 0.04 & 0.05 & 19 \\
\hline HIGMUF & 0.135 & 0.13 & 0.248 & 0.10 & 0.12 & 51 \\
\hline НМНТМС10 & 0.131 & 0.02 & 0.242 & 0.03 & 0.03 & 24 \\
\hline IMDIAC02 & 0.126 & 0.04 & 0.234 & 0.03 & 0.02 & 9 \\
\hline JACVAK & 0.134 & 0.07 & 0.232 & 0.07 & 0.05 & 38 \\
\hline JENMEU & 0.120 & 0.05 & 0.239 & 0.08 & 0.05 & $13(\mathrm{a})$ \\
\hline JIKCUB & 0.123 & 0.08 & 0.247 & 0.24 & 0.07 & 20 \\
\hline JITVAJ & 0.125 & 0.03 & 0.241 & 0.04 & 0.01 & 23 \\
\hline JOCCEJ & 0.102 & 0.02 & 0.250 & 0.03 & 0.04 & 15 \\
\hline JOCYAB & 0.118 & 0.09 & 0.249 & 0.11 & 0.04 & 23 \\
\hline JOPJUT & 0.121 & 0.08 & 0.243 & 0.09 & 0.06 & 32 \\
\hline JOYZIG & 0.144 & 0.02 & 0.236 & 0.09 & 0.06 & 18 \\
\hline JOZFIN & 0.113 & 0.05 & 0.240 & 0.05 & 0.06 & $42(a)$ \\
\hline JUBRON & 0.127 & 0.04 & 0.221 & 0.13 & 0.07 & 32 \\
\hline JUWJAM & 0.119 & 0.03 & 0.244 & 0.07 & 0.03 & 46 \\
\hline KABVUE & 0.127 & 0.04 & 0.245 & 0.20 & 0.08 & 41 \\
\hline KOTDEC & 0.134 & 0.02 & 0.248 & 0.01 & 0.02 & 19 \\
\hline KOZBAC & 0.123 & 0.04 & 0.238 & 0.03 & 0.05 & 19 \\
\hline KULBAU & 0.124 & 0.26 & 0.245 & 0.18 & 0.13 & 28 \\
\hline KUTJAK & 0.125 & 0.07 & 0.232 & 0.06 & 0.05 & 21 \\
\hline LENZEJ & 0.070 & 0.05 & 0.248 & 0.08 & 0.10 & 46 \\
\hline LERLAV & 0.030 & 0.02 & 0.249 & 0.01 & 0.01 & 14 \\
\hline
\end{tabular}




\begin{tabular}{|c|c|c|c|c|c|c|}
\hline LEZPAH & 0.125 & 0.03 & 0.246 & 0.01 & 0.02 & 16 \\
\hline LICXAW & 0.121 & 0.07 & 0.246 & 0.05 & 0.06 & 29 \\
\hline \multirow[t]{2}{*}{ MAPSIU } & 0.010 & 0.29 & 0.236 & 0.21 & 0.16 & 25 \\
\hline & 0.123 & 0.09 & 0.246 & 0.10 & 0.09 & 25 \\
\hline \multicolumn{7}{|l|}{ MEPHMN10 } \\
\hline MPRNLD & 0.106 & 0.19 & 0.249 & 0.17 & 0.29 & 26 \\
\hline MXZDEC & 0.097 & 0.04 & 0.250 & 0.03 & 0.04 & 13 \\
\hline NIPALA & 0.125 & 0.01 & 0.249 & 0.01 & 0.00 & 16 \\
\hline NMBZAX & 0.140 & 0.01 & 0.247 & 0.01 & 0.04 & 13 \\
\hline OTROSC & 0.118 & 0.01 & 0.248 & 0.02 & 0.05 & 14 \\
\hline PABNEL & 0.121 & 0.02 & 0.248 & 0.04 & 0.03 & 15 \\
\hline PARYUC & 0.128 & 0.06 & 0.247 & 0.08 & 0.08 & 22 \\
\hline PAZCBN01 & 0.016 & 0.05 & 0.237 & 0.06 & 0.04 & 12 \\
\hline PIPPTC10 & 0.124 & 0.03 & 0.241 & 0.04 & 0.02 & 15 \\
\hline PIYZAY & 0.127 & 0.05 & 0.244 & 0.09 & 0.05 & 42 \\
\hline PODHAR & 0.121 & 0.03 & 0.248 & 0.03 & 0.04 & 16 \\
\hline POFMUS & 0.126 & 0.19 & 0.250 & 0.22 & 0.10 & 18 \\
\hline PPHGFA & 0.120 & 0.38 & 0.245 & 0.14 & 0.08 & 20 \\
\hline PYRFUR & 0.146 & 0.02 & 0.235 & 0.01 & 0.02 & 19 \\
\hline SABTOE & 0.125 & 0.06 & 0.238 & 0.08 & 0.03 & 28 \\
\hline SAFNIW & 0.017 & 0.02 & 0.241 & 0.07 & 0.04 & 19 \\
\hline SAKLEV & 0.122 & 0.03 & 0.248 & 0.02 & 0.03 & 19 \\
\hline SEFBAG & 0.036 & 0.02 & 0.247 & 0.03 & 0.06 & 15 \\
\hline SEYXUP & 0.129 & 0.05 & 0.228 & 0.18 & 0.11 & $38(\mathrm{e})$ \\
\hline SIMZAP & 0.112 & 0.06 & 0.247 & 0.20 & 0.09 & 30 \\
\hline SITLAI & 0.061 & 0.18 & 0.246 & 0.16 & 0.14 & 34 \\
\hline SORFUA & 0.110 & 0.16 & 0.246 & 0.31 & 0.27 & 40 \\
\hline SOSVIF & 0.116 & 0.03 & 0.240 & 0.11 & 0.03 & 32 \\
\hline SUCGUS & 0.120 & 0.01 & 0.236 & 0.01 & 0.03 & 17 \\
\hline SUHHOS & 0.126 & 0.16 & 0.246 & 0.31 & 0.20 & 40 \\
\hline TAKHAO & 0.124 & 0.06 & 0.247 & 0.04 & 0.10 & 31 \\
\hline TEFHAN & 0.062 & 0.13 & 0.107 & 0.02 & 0.17 & 16 \\
\hline \multirow[t]{2}{*}{ TEJFUJ } & 0.109 & 0.08 & 0.248 & 0.08 & 0.06 & 41 \\
\hline & 0.124 & 0.05 & 0.238 & 0.24 & 0.09 & 19 \\
\hline \multicolumn{7}{|l|}{ TETCCU10 } \\
\hline TEWPAM & 0.143 & 0.11 & 0.245 & 0.12 & 0.08 & 44 \\
\hline TGLCAB10 & 0.127 & 0.04 & 0.249 & 0.03 & 0.04 & 18 \\
\hline TISZIE & 0.126 & 0.09 & 0.244 & 0.15 & 0.13 & 57 \\
\hline TIVSIA & 0.117 & 0.07 & 0.240 & 0.03 & 0.11 & 26 \\
\hline TOFXOB & 0.116 & 0.05 & 0.234 & 0.10 & 0.05 & 16 \\
\hline TOMYEZ & 0.134 & 0.02 & 0.249 & 0.01 & 0.05 & 31 \\
\hline TYRAMH & 0.009 & 0.12 & 0.245 & 0.02 & 0.04 & $10(e)$ \\
\hline VCZFCP & 0.122 & 0.11 & 0.001 & 0.09 & 0.09 & 35 \\
\hline VEJVOV & 0.124 & 0.06 & 0.237 & 0.13 & 0.10 & 19 \\
\hline VENRUB & 0.107 & 0.04 & 0.239 & 0.13 & 0.03 & 21 \\
\hline VETMOW & 0.127 & 0.04 & 0.244 & 0.08 & 0.05 & 23 \\
\hline VIFLAX & 0.151 & 0.03 & 0.249 & 0.01 & 0.02 & 29 \\
\hline VINVET & 0.125 & 0.02 & 0.233 & 0.03 & 0.04 & 22 \\
\hline VIPLOV & 0.124 & 0.08 & 0.249 & 0.09 & 0.11 & 37 \\
\hline VOKYAV & 0.120 & 0.03 & 0.244 & 0.03 & 0.04 & 19(e) \\
\hline
\end{tabular}




\begin{tabular}{|c|c|c|c|c|c|c|}
\hline VOSSEB & 0.130 & 0.05 & 0.246 & 0.08 & 0.09 & 43 \\
\hline VOVVAD & 0.124 & 0.02 & 0.250 & 0.02 & 0.02 & 25 \\
\hline VOZMIG & 0.074 & 0.04 & 0.248 & 0.03 & 0.05 & 53 \\
\hline VUDKAG & 0.121 & 0.05 & 0.235 & 0.13 & 0.05 & 25 \\
\hline WAKLAV & 0.132 & 0.04 & 0.246 & 0.15 & 0.06 & 15 \\
\hline \multirow[t]{2}{*}{ WAPPUY } & 0.125 & 0.06 & 0.199 & 0.11 & 0.08 & $24(\mathrm{c})$ \\
\hline & 0.125 & 0.07 & 0.247 & 0.25 & 0.02 & 24 \\
\hline WARYAP & 0.109 & 0.03 & 0.248 & 0.05 & 0.02 & 119 \\
\hline WEBBAG & 0.135 & 0.06 & 0.242 & 0.10 & 0.07 & 44 \\
\hline WELLUU & 0.126 & 0.11 & 0.005 & 0.15 & 0.15 & 27 \\
\hline WERNOW & 0.123 & 0.19 & 0.014 & 0.15 & 0.09 & 70 \\
\hline WIBLIC & 0.121 & 0.08 & 0.246 & 0.06 & 0.04 & 27 \\
\hline YAKNON & 0.124 & 0.07 & 0.247 & 0.08 & 0.06 & 44 \\
\hline YARDEA & 0.127 & 0.11 & 0.151 & 0.16 & 0.16 & 35 \\
\hline YEDROO & 0.127 & 0.06 & 0.056 & 0.11 & 0.09 & 24 \\
\hline YIMBUR & 0.002 & 0.07 & 0.248 & 0.08 & 0.08 & 22 Pbca? \\
\hline YITMOD & 0.121 & 0.02 & 0.247 & 0.04 & 0.02 & 18 \\
\hline YIVGEP & 0.127 & 0.05 & 0.243 & 0.06 & 0.06 & 47 \\
\hline YOJJEM & 0.242 & 0.26 & 0.144 & 0.10 & 0.25 & 31 \\
\hline YOKJOX & 0.159 & 0.02 & 0.250 & 0.02 & 0.03 & 24 \\
\hline YOLWIF & 0.125 & 0.05 & 0.249 & 0.04 & 0.04 & 31 \\
\hline YOPTAY & 0.123 & 0.08 & 0.234 & 0.26 & 0.08 & 27 \\
\hline YOYXEP & 0.172 & 0.05 & 0.240 & 0.06 & 0.04 & 17 \\
\hline YUCTIZ & 0.092 & 0.09 & 0.244 & 0.03 & 0.10 & 35 \\
\hline YUGRUN & 0.005 & 0.06 & 0.249 & 0.13 & 0.26 & 20 Pbca? \\
\hline ZAJGEW & 0.137 & 0.03 & 0.247 & 0.07 & 0.05 & 20 \\
\hline \multirow[t]{2}{*}{ ZAYKAL } & 0.125 & 0.07 & 0.231 & 0.08 & 0.05 & $25(\mathrm{c})$ \\
\hline & 0.125 & 0.22 & 0.208 & 0.14 & 0.08 & 25 \\
\hline ZEQHIM & 0.129 & 0.43 & 0.210 & 0.11 & 0.34 & 15 \\
\hline ZEXSEA & 0.118 & 0.07 & 0.250 & 0.05 & 0.06 & 52 \\
\hline ZICGOH & 0.117 & 0.08 & 0.246 & 0.08 & 0.09 & 27 \\
\hline ZIXHET & 0.125 & 0.11 & 0.235 & 0.06 & 0.12 & 42 \\
\hline ZOGGIM & 0.118 & 0.32 & 0.236 & 0.08 & 0.32 & 61 (a) \\
\hline ZOLZAB & 0.124 & 0.07 & 0.213 & 0.06 & 0.03 & 29 \\
\hline ZORTEF & 0.126 & 0.04 & 0.233 & 0.05 & 0.03 & 25 \\
\hline ZOSXEK & 0.119 & 0.05 & 0.208 & 0.05 & 0.05 & 25 \\
\hline ZUFWIG & 0.007 & 0.01 & 0.241 & 0.01 & 0.02 & 9 Pbca? \\
\hline \multirow[t]{2}{*}{ ZUXXAT } & 0.011 & 0.03 & 0.249 & 0.02 & 0.07 & 23 Pbca? \\
\hline & 0.127 & 0.02 & 0.249 & 0.03 & 0.05 & 16 \\
\hline
\end{tabular}

ZZZMUC01
(a) Some disordered atoms omitted from pairing.
(b) Space group given as $P b c 2{ }_{1}$; probably should be $P c 2{ }_{1} b$
(c) There are two approximate centers.
(d) The lengths of the $b$ and $c$ axes are probably interchanged.
(e) One unpaired water molecule omitted. 
Table 2. Space group Pna ${ }_{1}$ : 169 examples of crystals (identified by their REFCODES) which have local centers of symmetry (156 with $Z=8$ and 13 with $Z=4$ ). As noted in the text, the mean $\mathrm{x}$ and y coordinates of the local centers have been adjusted to lie within the limits $0^{2} x, y^{2} 1 / 4$, with $z=0$. 'rmsd' is defined in the caption to Table 1.

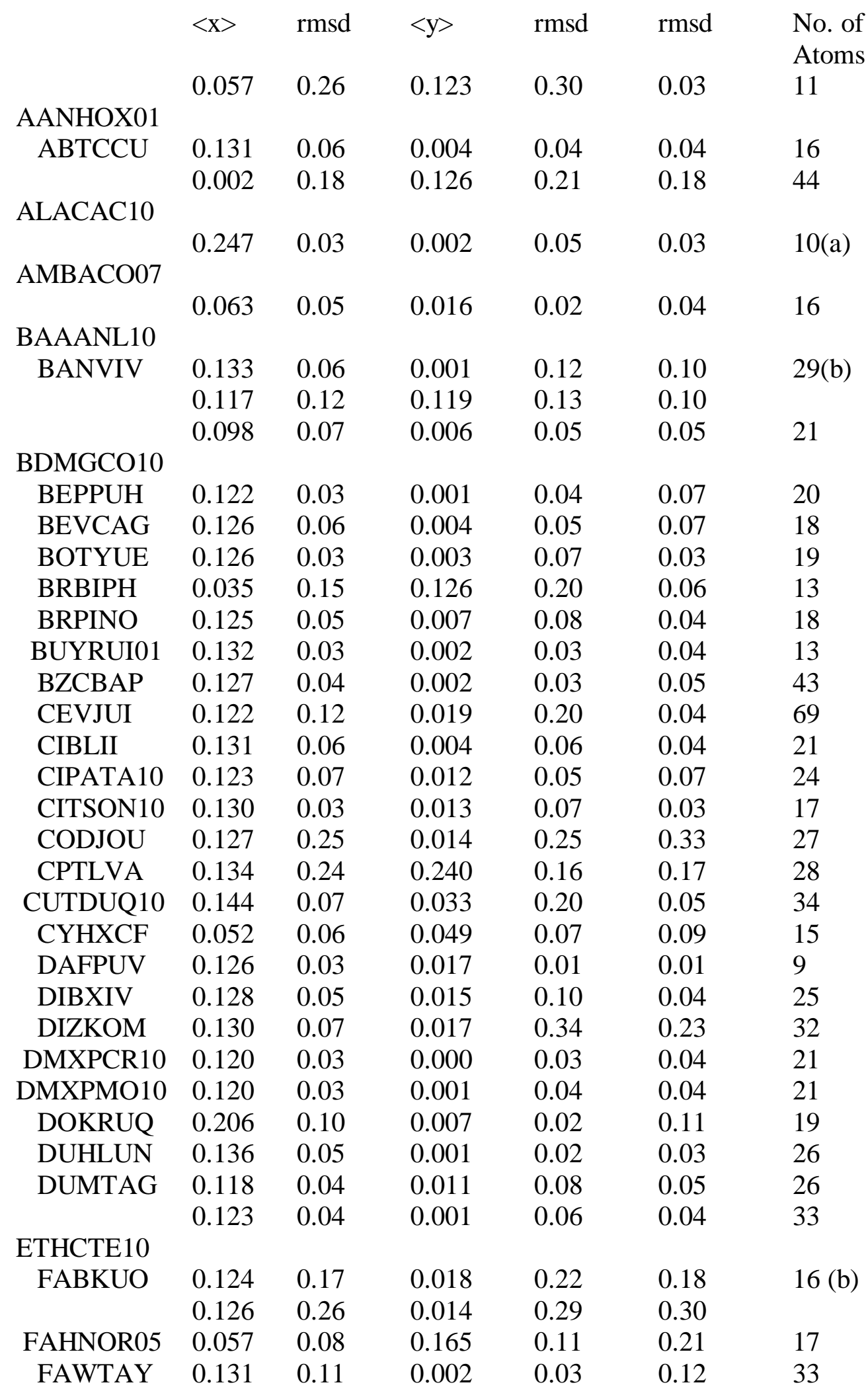




\begin{tabular}{|c|c|c|c|c|c|}
\hline FAYGAN01 & 0.128 & 0.07 & 0.008 & 0.11 & 0.04 \\
\hline FETCOW & 0.134 & 0.10 & 0.145 & 0.11 & 0.07 \\
\hline FIRYIO & 0.124 & 0.04 & 0.002 & 0.03 & 0.04 \\
\hline FISFES & 0.185 & 0.04 & 0.008 & 0.05 & 0.02 \\
\hline FOFXED & 0.125 & 0.18 & 0.054 & 0.23 & 0.18 \\
\hline FOPMOM & 0.151 & 0.10 & 0.002 & 0.04 & 0.04 \\
\hline FULLAZ & 0.126 & 0.03 & 0.007 & 0.01 & 0.02 \\
\hline FUYMIV & 0.120 & 0.03 & 0.247 & 0.04 & 0.04 \\
\hline GAFHAW & 0.168 & 0.28 & 0.052 & 0.27 & 0.94 \\
\hline GAFNOQ & 0.136 & 0.03 & 0.207 & 0.04 & 0.09 \\
\hline GAXJIY & 0.120 & 0.01 & 0.002 & 0.03 & 0.03 \\
\hline GCVINC10 & 0.117 & 0.21 & 0.229 & 0.08 & 0.15 \\
\hline GESCAI & 0.114 & 0.11 & 0.016 & 0.04 & 0.08 \\
\hline GIKDIN & 0.126 & 0.03 & 0.004 & 0.07 & 0.07 \\
\hline GINCAH & 0.125 & 0.06 & 0.233 & 0.06 & 0.11 \\
\hline HABJUP01 & 0.249 & 0.02 & 0.126 & 0.02 & 0.00 \\
\hline HACNAQ & 0.123 & 0.03 & 0.002 & 0.04 & 0.05 \\
\hline HAZWOU & 0.125 & 0.30 & 0.004 & 0.12 & 0.17 \\
\hline HEWKEZ & 0.120 & 0.03 & 0.004 & 0.03 & 0.06 \\
\hline HIFSUK & 0.122 & 0.10 & 0.022 & 0.07 & 0.12 \\
\hline HIHLAL & 0.132 & 0.05 & 0.018 & 0.04 & 0.05 \\
\hline HMESBB & 0.193 & 0.11 & 0.032 & 0.05 & 0.11 \\
\hline НХМНСН10 & 0.117 & 0.04 & 0.248 & 0.02 & 0.02 \\
\hline IMZNCU & 0.126 & 0.06 & 0.003 & 0.10 & 0.09 \\
\hline JEGDEE & 0.127 & 0.05 & 0.004 & 0.05 & 0.05 \\
\hline JEWBIX & 0.127 & 0.02 & 0.000 & 0.03 & 0.02 \\
\hline JEZSOW & 0.115 & 0.21 & 0.007 & 0.22 & 0.36 \\
\hline JIRDOD & 0.120 & 0.01 & 0.002 & 0.01 & 0.02 \\
\hline JISVEM & 0.007 & 0.19 & 0.234 & 0.11 & 0.14 \\
\hline JOFHIV & 0.125 & 0.00 & 0.001 & 0.01 & 0.01 \\
\hline JOPYES & 0.128 & 0.28 & 0.015 & 0.04 & 0.37 \\
\hline JOXDAB & 0.113 & 0.02 & 0.002 & 0.01 & 0.02 \\
\hline JOXLOX & 0.125 & 0.04 & 0.014 & 0.03 & 0.07 \\
\hline JUJXUH & 0.123 & 0.05 & 0.243 & 0.07 & 0.05 \\
\hline JUJYAO & 0.124 & 0.07 & 0.242 & 0.07 & 0.04 \\
\hline JULNUZ & 0.118 & 0.07 & 0.003 & 0.06 & 0.08 \\
\hline JUNFUT & 0.119 & 0.04 & 0.003 & 0.05 & 0.05 \\
\hline $\mathrm{KACDOH}$ & 0.125 & 0.16 & 0.038 & 0.14 & 0.16 \\
\hline KAJPIU & 0.243 & 0.23 & 0.126 & 0.24 & 0.32 \\
\hline KEDDIG & 0.125 & 0.04 & 0.001 & 0.03 & 0.04 \\
\hline KELLUI & 0.129 & 0.08 & 0.006 & 0.07 & 0.08 \\
\hline KEVFUM & 0.125 & 0.04 & 0.002 & 0.02 & 0.02 \\
\hline KILWOR & 0.232 & 0.14 & 0.124 & 0.08 & 0.08 \\
\hline KOCYIK & 0.129 & 0.02 & 0.001 & 0.03 & 0.05 \\
\hline KOVXAU & 0.126 & 0.11 & 0.020 & 0.08 & 0.04 \\
\hline KOYHEL & 0.128 & 0.03 & 0.002 & 0.04 & 0.04 \\
\hline KUFHIC & 0.144 & 0.03 & 0.004 & 0.06 & 0.07 \\
\hline KUNXEW & 0.125 & 0.17 & 0.161 & 0.03 & 0.17 \\
\hline LAMBAC & 0.127 & 0.07 & 0.009 & 0.08 & 0.09 \\
\hline MAMBOR & 0.114 & 0.03 & 0.031 & 0.06 & 0.03 \\
\hline
\end{tabular}




\begin{tabular}{|c|c|c|c|c|c|c|}
\hline MAMBRF & 0.129 & 0.08 & 0.006 & 0.05 & 0.08 & 10 \\
\hline МЕНВРН & 0.132 & 0.17 & 0.018 & 0.31 & 0.08 & 20 \\
\hline NMPCPA10 & 0.132 & 0.02 & 0.010 & 0.09 & 0.04 & 13 \\
\hline PATZEP & 0.126 & 0.04 & 0.002 & 0.04 & 0.03 & 23 \\
\hline PEZZOJ & 0.116 & 0.02 & 0.002 & 0.11 & 0.11 & 39 \\
\hline PFEPPT & 0.191 & 0.04 & 0.248 & 0.04 & 0.08 & 37( \\
\hline PHTHFF & 0.113 & 0.04 & 0.001 & 0.04 & 0.06 & 34 \\
\hline PIPPAF & 0.126 & 0.10 & 0.101 & 0.07 & 0.09 & 28 \\
\hline POBWEI & 0.123 & 0.15 & 0.027 & 0.32 & 0.12 & 25 \\
\hline POMGIH & 0.126 & 0.07 & 0.007 & 0.07 & 0.06 & 23 \\
\hline \multirow[t]{2}{*}{ PORLOX } & 0.125 & 0.05 & 0.023 & 0.05 & 0.04 & 23 \\
\hline & 0.032 & 0.31 & 0.123 & 0.23 & 0.03 & $9(b)$ \\
\hline \multicolumn{7}{|l|}{ PYRZOL02 } \\
\hline & 0.022 & 0.26 & 0.237 & 0.27 & 0.05 & \\
\hline & 0.127 & 0.18 & 0.015 & 0.22 & 0.26 & 16 \\
\hline \multicolumn{7}{|l|}{ QQQCAS01 } \\
\hline SAZCAX & 0.134 & 0.04 & 0.002 & 0.04 & 0.11 & 22 \\
\hline SEPPIM & 0.127 & 0.04 & 0.003 & 0.02 & 0.03 & 27 \\
\hline SIKRIN & 0.125 & 0.05 & 0.005 & 0.05 & 0.05 & 20 \\
\hline SIWDEH01 & 0.102 & 0.04 & 0.001 & 0.05 & 0.03 & 25 \\
\hline \multirow[t]{2}{*}{ SIXZEE } & 0.123 & 0.16 & 0.017 & 0.21 & 0.17 & 16 \\
\hline & 0.113 & 0.01 & 0.002 & 0.02 & 0.05 & 18 \\
\hline \multicolumn{7}{|l|}{ SLFNMA02 } \\
\hline SOYCUE & 0.127 & 0.06 & 0.033 & 0.11 & 0.09 & 27 \\
\hline SUCHED & 0.127 & 0.03 & 0.001 & 0.03 & 0.03 & 18 \\
\hline SUPMUL & 0.128 & 0.02 & 0.005 & 0.07 & 0.07 & 54 \\
\hline TACQOD & 0.128 & 0.16 & 0.003 & 0.16 & 0.20 & 30 \\
\hline TCENCU & 0.156 & 0.17 & 0.118 & 0.20 & 0.09 & 11 \\
\hline TCXCPD & 0.129 & 0.03 & 0.247 & 0.05 & 0.02 & 16 \\
\hline \multirow[t]{2}{*}{ TEPWAM } & 0.121 & 0.21 & 0.007 & 0.20 & 0.16 & 82 \\
\hline & 0.127 & 0.06 & 0.013 & 0.16 & 0.09 & 17 \\
\hline \multicolumn{7}{|l|}{ THUPDC01 } \\
\hline TIQLIO & 0.043 & 0.18 & 0.013 & 0.17 & 0.17 & 47 \\
\hline TIVWUQ & 0.120 & 0.04 & 0.001 & 0.05 & 0.06 & 21 \\
\hline TMPSUL10 & 0.126 & 0.04 & 0.003 & 0.02 & 0.02 & 18 \\
\hline TOJRIT & 0.125 & 0.01 & 0.005 & 0.04 & 0.11 & 25 \\
\hline TOLDIN & 0.128 & 0.03 & 0.012 & 0.08 & 0.04 & 8 \\
\hline TORDAZ & 0.126 & 0.13 & 0.027 & 0.19 & 0.15 & 25 \\
\hline TOSCEJ & 0.131 & 0.08 & 0.010 & 0.11 & 0.04 & 35 \\
\hline TOYKEX & 0.123 & 0.03 & 0.001 & 0.03 & 0.04 & 81 \\
\hline TUDQAK & 0.112 & 0.04 & 0.001 & 0.04 & 0.05 & 56 \\
\hline TUKWOL & 0.129 & 0.02 & 0.247 & 0.02 & 0.01 & 6 \\
\hline TUKYUT & 0.125 & 0.03 & 0.231 & 0.02 & 0.10 & 35 \\
\hline TUKZAA & 0.125 & 0.05 & 0.232 & 0.05 & 0.03 & 71 \\
\hline VABBIJ & 0.134 & 0.03 & 0.001 & 0.01 & 0.02 & 17 \\
\hline VALWOU & 0.124 & 0.02 & 0.055 & 0.04 & 0.04 & 18 \\
\hline VATZUL & 0.093 & 0.14 & 0.231 & 0.24 & 0.01 & 19 \\
\hline VAVKEI & 0.126 & 0.06 & 0.014 & 0.04 & 0.10 & 21 \\
\hline VEKTOU & 0.120 & 0.02 & 0.243 & 0.05 & 0.03 & 26 \\
\hline VEZJIT10 & 0.146 & 0.10 & 0.001 & 0.10 & 0.07 & 40 \\
\hline
\end{tabular}




$\begin{array}{lllllll}\text { VIGZUG } & 0.129 & 0.08 & 0.010 & 0.06 & 0.11 & 28 \\ \text { VINCBZ } & 0.130 & 0.42 & 0.006 & 0.03 & 0.40 & 15 \\ \text { VOVRED } & 0.125 & 0.06 & 0.004 & 0.21 & 0.16 & 26 \\ \text { VOVTEF } & 0.128 & 0.02 & 0.006 & 0.05 & 0.03 & 26 \\ \text { VOYJUO } & 0.130 & 0.03 & 0.246 & 0.04 & 0.05 & 35 \\ \text { VUPRIH } & 0.169 & 0.15 & 0.006 & 0.05 & 0.22 & 28 \\ \text { WAHGAN } & 0.190 & 0.05 & 0.002 & 0.09 & 0.05 & 46 \\ \text { WAMXAJ } & 0.127 & 0.03 & 0.212 & 0.13 & 0.13 & 35 \\ \text { WARZIY } & 0.130 & 0.07 & 0.001 & 0.05 & 0.03 & 54 \\ \text { WAVGEF } & 0.127 & 0.04 & 0.001 & 0.06 & 0.08 & 29 \\ \text { WAXYAV } & 0.128 & 0.03 & 0.249 & 0.04 & 0.04 & 38 \\ \text { WEGPON } & 0.126 & 0.02 & 0.028 & 0.04 & 0.04 & 7 \\ \text { WEGVIN } & 0.129 & 0.06 & 0.001 & 0.05 & 0.04 & 49 \\ & 0.132 & 0.11 & 0.036 & 0.23 & 0.23 & 23 \\ \text { WEMWUG } & & & & & & \\ \text { WIMBUP } & 0.149 & 0.08 & 0.002 & 0.06 & 0.06 & 58 \\ \text { WINTES } & 0.118 & 0.04 & 0.015 & 0.09 & 0.04 & 36 \\ \text { YAYMUG } & 0.128 & 0.05 & 0.021 & 0.04 & 0.05 & 37 \\ \text { YAYVOJ } & 0.124 & 0.07 & 0.009 & 0.07 & 0.08 & 35 \\ \text { YELSOX } & 0.125 & 0.03 & 0.010 & 0.08 & 0.06 & 9 \\ \text { YELSUD } & 0.123 & 0.04 & 0.011 & 0.09 & 0.08 & 9 \\ \text { YENREO } & 0.124 & 0.03 & 0.011 & 0.12 & 0.02 & 19 \\ \text { YERHEI } & 0.114 & 0.04 & 0.003 & 0.04 & 0.05 & 20 \\ \text { YEWDEJ } & 0.126 & 0.04 & 0.007 & 0.05 & 0.04 & 21 \\ \text { YORLEW } & 0.121 & 0.10 & 0.000 & 0.07 & 0.04 & 21 \\ \text { YUJGEP } & 0.095 & 0.05 & 0.001 & 0.03 & 0.03 & 40 \\ \text { YUJLEU01 } & 0.123 & 0.06 & 0.019 & 0.04 & 0.05 & 21 \\ \text { YUKWEG } & 0.131 & 0.02 & 0.001 & 0.03 & 0.04 & 63 \\ \text { YUPTUY } & 0.124 & 0.08 & 0.003 & 0.06 & 0.09 & 24 \\ \text { ZAJVAH } & 0.124 & 0.12 & 0.021 & 0.15 & 0.13 & 26 \\ \text { ZANJON } & 0.125 & 0.05 & 0.015 & 0.06 & 0.06 & 30 \\ \text { ZASMIP } & 0.125 & 0.01 & 0.013 & 0.19 & 0.02 & 18 \\ \text { ZEQBUS } & 0.123 & 0.03 & 0.004 & 0.03 & 0.03 & 41 \\ \text { ZIPPOD } & 0.122 & 0.04 & 0.000 & 0.02 & 0.03 & 25 \\ \text { ZOCFUS } & 0.121 & 0.14 & 0.003 & 0.08 & 0.14 & 34 \\ \text { ZOGQAN01 } & 0.128 & 0.07 & 0.002 & 0.03 & 0.02 & 37 \\ \text { ZOGRAO } & 0.125 & 0.02 & 0.002 & 0.02 & 0.09 & 18 \\ \text { ZOLRIB } & 0.187 & 0.26 & 0.013 & 0.13 & 0.36 & 15 \\ \text { ZOWSAF } & 0.121 & 0.02 & 0.002 & 0.04 & 0.03 & 32 \\ \text { ZUWVIW } & 0.113 & 0.10 & 0.013 & 0.08 & 0.02 & 18 \\ & 0.139 & 0.02 & 0.004 & 0.02 & 0.02 & 16\end{array}$

ZZZOVA01
(a) The two related molecules are slightly different, one being a zwitterion (see text).
(b) There are two approximate centers in this structure.
(c) A dichloromethane solvent molecule is unpaired, and has been omitted. 